\title{
Quantitative Measurements of Cerebral Blood Flow in Patients With Unilateral Carotid Artery Occlusion: A PET and MR Study
}

\author{
Weili Lin, PhD ${ }^{1,{ }^{*}}$, Azim Celik, $\mathrm{MS}^{2}$, Colin Derdeyn, $\mathrm{MD}^{3}$, Hongyu An, $\mathrm{MS}^{4}$, Yueh Lee, MS ${ }^{1}$, \\ Tom Videen, $\mathbf{P h D}^{5}$, Leif Ostergaard, $\mathbf{M D}^{6}$, and William J. Powers, $\mathbf{M D}^{3,5}$ \\ ${ }^{1}$ Department of Radiology, University of North Carolina at Chapel Hill, Chapel Hill, North Carolina \\ ${ }^{2}$ Department of Electrical Engineering, Washington University, St. Louis, Missouri \\ ${ }^{3}$ Department of Radiology, Washington University, St. Louis, Missouri \\ ${ }^{4}$ Department of Biomedical Engineering, Washington University, St. Louis, Missouri \\ ${ }^{5}$ Department of Neurology, Washington University, St. Louis, Missouri \\ ${ }^{6}$ Department of Neuroradiology, Aarhus University Hospital, Aarhus, Denmark
}

\begin{abstract}
Although it has been demonstrated that quantitative measures of cerebral blood flow (CBF) can be obtained with the singular value decomposition (SVD) algorithm, the extent to which quantitative CBF measurements can be utilized under pathophysiological conditions has not been systematically studied. A total of five healthy volunteers and five patients with unilateral carotid artery occlusion were studied. Only magnetic resonance (MR) images were acquired for the volunteer group while both MR and positron emission tomography (PET) images were acquired for the patient group. Assessments of CBF from normal volunteers compared favorably with values reported in the literature. However, while a linear relationship was observed for each patient when MR measured CBF was compared to that obtained from PET, this linear relationship diminished when all patients were analyzed as a group $(r=0.41)$. A correction factor $(\mathrm{CF})$ was proposed that was equal to the ratio of the area of the venous output function (VOF) in each patient to the mean VOF obtained from the volunteer group. After globally scaling the CBF of each patient based on the experimentally derived CF, a substantial improvement was observed (a slope of 1.02 and $r=0.8$ for the linear regression line) in the relationship between MR estimated CBF and those obtained from PET.
\end{abstract}

\section{Index terms}

cerebral blood flow; cerebrovascular disease; perfusion imaging; PET; MRI

\footnotetext{
(C) 2001 Wiley-Liss, Inc.

*Address reprint requests to:W.L., University of North Carolina at Chapel Hill, Department of Radiology, CB\#7515, Chapel Hill, NC 27599.weili_lin@med.unc.edu.

This paper was presented at the 2001 ISMRM meeting, p106.
} 
Alterations Of Cerebral Hemodynamics are commonly associated with cerebrovascular disease. Therefore, an accurate, non-invasive, and accessible method of measuring cerebral blood flow (CBF) may elucidate our understanding of the cerebrovascular disorders. Positron emission tomography (PET) provides accurate, quantitative, and repeatable in vivo measurements of CBF in humans. These measurements have both confirmed the presence of known cerebrovascular physiologic and pathologic mechanisms and yielded new information about cerebral hemodynamics (1). However, PET is relatively expensive, physician intensive, and not widely available. In contrast, with the advent of fast imaging techniques, magnetic resonance imaging (MRI) now has the potential to characterize rapidly changing physiologic and pathophysiologic states, including those related to changes in tissue perfusion (2-9). However, only relative measurements are available for many of these techniques and they have not yet been systematically validated with respect to relevant physiologic variables.

It has been shown that an estimate of cerebral hemo-dynamics including CBF and cerebral blood volume (CBV) can be obtained with a dynamic MR imaging approach (2-9). With a singular value decomposition (SVD) deconvolution approach, Ostergaard, et al (10-13) demonstrated that an estimate of the residue function can be obtained, which in turn provides a measure of CBF. In order to assess the accuracy of this approach, they compared the MR estimated CBF to those obtained from PET in animals under both normocapnic and hypercapnic conditions (12). A highly linear relationship was obtained between the MR estimates and PET measurements over a physiologically relevant range of CBF. In addition, results from volunteer studies demonstrated that the MR estimated CBF compared favorably with the known CBF values in the literature within individuals (13). Therefore, they concluded that an accurate estimate of CBF in humans could be achieved with the SVD approach.

However, to a large extent, the quantitative aspects of SVD have only been investigated in normal volunteers and relatively little attention has been given to whether a consistent estimate of CBF can be obtained under pathophysiological conditions. In this study, normal volunteers and patients with unilateral carotid artery occlusion were studied. In the patient group, CBF estimates were obtained from both PET and MR so that comparisons between MR estimated CBF and that obtained from PET in the same patient could be assessed under a pathological condition. In contrast, only MR scans were performed in the volunteer group. A correction scheme was proposed to minimize the variability and inconsistency for the MR measurements of $\mathrm{CBF}$ in the patient group. Results on the effectiveness of the proposed correction scheme will be demonstrated.

\section{MATERIALS AND METHODS}

\section{Subject Information}

In total, five healthy volunteers and five patients with unilateral carotid artery occlusion were studied. All patients were recruited from the St. Louis Carotid Occlusion Study $(14,15)$, which is a longitudinal study of hemodynamic factors in patients with symptomatic and asymptomatic carotid artery occlusion. For the patient group, both PET and MR images were acquired on the same day, while only MR studies were performed for the volunteer 
group. The patient studies were approved by the Human Studies Committee at Washington University, while the volunteer studies were approved by the Human Studies Committee at the University of North Carolina at Chapel Hill. In all cases, written informed consent was obtained from all subjects.

\section{PET Imaging}

All PET studies were performed on a Siemens model 961 ECAT EXACT HR47 PET scanner. Subjects were supine on a specially designed couch in a room with low ambient noise. Both arterial and venous catheters were placed to permit the collection of arterial samples and the intravenous injection of radiotracers for PET studies and contrast agent for MR studies, respectively. A radial artery cannula was placed percutaneously after insuring that ulnar collateral circulation to the wrist was adequate by the Allen test. Subjects were then positioned in the PET scanner with the aid of a vertical laser line. The position of the patient's head relative to the plane of the PET scan was recorded by a lateral skull film marked with a radio-opaque line. An individually molded thermoplastic face mask was fitted to each subject to insure that the head remained in the same position throughout the study. Calibration of the PET scan to permit conversion of PET counts to quantitative radiotracer concentrations was carried out as previously described (16). Each subject underwent a transmission scan with gallium 68-germanium 68 rod sources in order to provide attenuation data necessary for the quantitative reconstruction of subsequent scans. CBF was measured by an adaptation of the Kety autoradiographic method using a bolus intravenous injection of oxygen-15 labeled water (16-18). Arterial blood was drawn to generate an arterial timeactivity curve of radiotracer concentration. All radiotracers were produced in the Washington University Medical Cyclotrons (19).

\section{MR Imaging}

All images were acquired on a Siemens 1.5-T VISION whole body clinical scanner (Siemens Medical Inc., Erlangen, Germany) with a maximum gradient strength of $25 \mathrm{mT} / \mathrm{m}$ and a ramp time of $0.6 \mathrm{msec}$. A standard circularly polarized head coil was used as the transmit/receive coil.

All subjects were supine. After obtaining scout images, conventional anatomical images including spin density, T1-, and T2-weighted images were obtained. In addition, a radiofrequency spoiled three-dimensional FLASH sequence was used to obtain threedimensional T1-weighted images, which covered the entire brain. Velocity compensation along both the slice select and frequency encoding directions was used to minimize flow artifacts. The imaging parameters were as follows: TR $=20 \mathrm{msec}$; TE $=6 \mathrm{msec}$; flip angle $(\mathrm{FA})=25^{\circ}$; slice thickness $(\mathrm{TH})=2-2.5 \mathrm{~mm}$ with 50-54 partitions to cover the whole brain; and in-plane resolution $=0.84 \times 0.84 \mathrm{~mm}^{2}$. Subsequently, a two-dimensional, singleshot gradient echo, echo planar imaging (EPI) sequence was utilized for acquiring images. The imaging parameters were as follows: $\mathrm{TR}=2$ seconds; $\mathrm{TE}=54 \mathrm{msec} ; \mathrm{TH}=6 \mathrm{~mm}$ with 12 slices; in-plane resolution $=1.8 \times 1.1 \mathrm{~mm}^{2}$; and $\mathrm{FA}=60^{\circ}$. This sequence was repeated 40 times while the subjects were lying still inside the MR scanner. A contrast agent $(0.1$ $\mathrm{mmol} / \mathrm{kg}$, Gd-DTPA) was administered intravenously at the completion of the fifth scan. 
CBF Estimates From MR Images-All images were transferred to a Sun Workstation (Sun Microsystems, Mountain View, CA) for post-processing. Detailed descriptions of the post-processing procedures for obtaining a quantitative estimate of $\mathrm{CBF}$ have been addressed elsewhere (10-13). In short, the MR acquired images were first converted to the $\Delta \mathrm{R} 2 *$ maps via

$$
\Delta \mathrm{R} 2^{*}=\ln (\mathrm{S}(0) / \mathrm{S}(\mathrm{t})) / \mathrm{TE} \quad(1)
$$

where $S(0)$ is the baseline signal obtained from the mean signal of the first 10 scans while $S(t)$ represents the signal of the acquired MR images as a function of time. Subsequently, $\Delta \mathrm{R} 2 *$ maps covering the Circle of Willis were examined and one pixel exhibiting maximal $\Delta \mathrm{R} 2 *$ and within the main segment of the middle cerebral artery or the posterior cerebral artery in the hemisphere contralateral to the occluded carotid artery was selected for obtaining the arterial input function (AIF). In order to remove the effects of recirculation of the contrast agent, pixel-by-pixel $\Delta \mathrm{R}^{*}$ time curve was fitted to an exponential function. Finally, SVD (10-13) was employed to deconvolve the tissue function (or curve) by the experimentally measured AIF via

$$
\mathrm{C}(\mathrm{t})=\mathrm{CBF}^{*}(\mathrm{AIF}(\mathrm{t}) \otimes \mathrm{R}(\mathrm{t}))
$$

where $\mathrm{R}(\mathrm{t})$ is the normalized residue function. Thus, a quantitative estimate of pixel-by-pixel CBF map could be obtained in vivo.

\section{Estimates of Venous Output Function (VOF)}

In addition to defining an AIF for SVD, a pixel within the superior sagittal sinus was also chosen to obtain an estimate of the area of VOF for both the patient and volunteer groups. The superior sagittal sinus was an easily identified structure even with the spatial resolution employed for the EPI sequences. In order to obtain the measurement of the area of VOF, a three-point moving average was applied pixel-by-pixel first to minimize variation of the signal time curves. Since the presence of contrast agent led to a signal reduction in MR gradient echo EPI images, a threshold of $\mathrm{S}(0)-2 * \mathrm{SD}$ was employed to determine the bolus arrival time $\left(\mathrm{T}_{\mathrm{a}}\right)$ and the time that the first pass of contrast agent finished passing through the imaging slices $\left(\mathrm{T}_{\text {end }}\right)$. The $\mathrm{SD}$ corresponds to the signal variation of the first 10 time points. The $T_{a}$ and $T_{\text {end }}$ were defined as the first and the last time points of the signal time curve having a signal intensity less than the predefined threshold, respectively. In contrast, the time point with the lowest signal intensity and between $T_{a}$ and $T_{\text {end }}$ was defined as $T_{0}$. After determining the three time points, concentration time curves $[\mathrm{C}(\mathrm{t})]$ were employed. In order to minimize the effects of secondary circulation of the contrast agent, a inflection time point $\left(T_{i n}\right)$ was obtained by taking the time derivative of $C(t)$ for $t>T_{0}$. When a sign change was detected, this time point was defined as $T_{i n}$. Subsequently, the exponential fitting was performed with the time points between $\mathrm{C}\left(\mathrm{T}_{\text {end }}-6\right)$ to $\mathrm{C}\left(\mathrm{T}_{\text {end }}\right)$ or $\mathrm{C}\left(\mathrm{T}_{\text {in }}\right)$ depending on which time point occurred earlier. Finally, the area of the VOF was obtained by integrating the exponentially fitted concentration time curves from $T_{a}$ to $T_{\text {end }}$ or $T_{\text {in }}$ with the same criterion. 
Correction of rCBF Measurements in Patients-Assuming that the VOF is free from partial volume effects with the surrounding brain parenchyma, the injected dose per $\mathrm{kg}$ of the contrast agent is constant among the subjects, and the fraction of cardiac output that goes to the brain is constant, the area of VOF should be identical among subjects. Therefore, a correction factor (CF), in theory, can be obtained for each patient by taking the ratio of the area of the VOF from each patient to the mean area of the VOF $\left(\mathrm{VOF}_{\text {mean }}\right)$ obtained from the volunteer studies. This approach was employed in our study to obtain a CF for each patient, and subsequently utilized to scale MR estimated CBF maps globally for each patient.

Co-registration-The following procedures were only done for patient studies since the PET was not performed for the volunteer group. All PET CBF maps were first converted to the Talairach space (20). A three-dimensional Guassian filter with a point spread function of $15 \mathrm{~mm}$ (full width half maximum [FWHM]) was applied to the acquired MR three-

dimensional T1-weighted images. By so doing, a comparable spatial resolution between the MR and PET images could be achieved. In addition, manually tracing via Analyze (Mayo Clinic, Rochester, MN) was employed to remove background, as well as the skull, signal from the T1-weighted images. This procedure was necessary to improve the accuracy of the subsequent co-registration between the MR and PET results. The filtered three-dimensional MR images were then co-registered with the PET CBF images via the Automated Image Registration (AIR) program (21) with the assumption of a rigid body motion. Finally, the rotational matrix obtained from the AIR program (21) was applied to the MR CBF maps.

Regional Analysis of CBF-In order to compare the CBF estimates obtained from both the MR and PET studies, a region of interest (ROI) analysis was performed. A total of 12 circular ROIs with three ROIs equally dividing each hemisphere across two adjacent slices were pre-defined. These ROIs were placed in both the PET and MR CBF maps and were used to obtain regional estimates of CBF in the patient group. In contrast, for the volunteer group, a total of 20 ROIs were defined across five slices with 10 ROIs placed in the gray matter and the remaining 10 ROIs located in the white matter, respectively. Mean and standard deviation from all ROIs were recorded for comparison.

Statistical Analysis-Data are expressed as mean \pm SD, where SD represents the intersubject variability. A linear regression analysis was used and a correlation coefficient ( $r$ ) was calculated to indicate the correlation between two experimentally measured parameters. A single factor analysis of variance (ANOVA) was also used for statistical analysis between the two groups. A $P$ value less than 0.05 was considered as a significant difference between groups at a $95 \%$ confidence level.

\section{RESULTS}

$\Delta \mathrm{R} 2 *$ time curves obtained from one pixel placed in the middle cerebral artery and one pixel located within the superior sagittal sinus of all volunteers are shown in Figure 1a and Figure $1 b$, respectively. The arrival times for both the AIF and VOF from all volunteers were adjusted to be identical so that a comparison of the shape, as well as the distributions, among subjects could be made. The experimentally measured AIFs exhibited a substantial 
variability from subject to subject, while a more consistent pattern was observed for the VOF among subjects. Furthermore, when the areas of the AIF and VOF were compared (Table 1), the area of the VOF was always larger than that of the AIF. A mean area of 509.4 \pm 107.3 was obtained for the AIF, while a mean area of $653.8 \pm 62.6(P<0.02)$ was obtained from all subjects, respectively. These findings suggest that the area of AIF may be reduced by partial volume effects with the surrounding tissue, causing the observed variability among subjects, as well as the reduced area, when compared to that of the VOF. The experimentally measured $\mathrm{VOF}_{\text {mean }}$ (Table 1 ) is subsequently utilized to derive the $\mathrm{CF}$ for each patient. The areas of AIF and VOF for the patient group are also given in Table 1. Similar findings to those observed in the volunteer group are seen. All areas obtained from the VOF were greater than the AIF. However, the inter-subject variability was much higher for both the AIF and VOF when compared to those obtained in the volunteer group. In addition, the areas of VOF of the patient group were larger than those of the volunteer group.

Representative MR measured CBF maps from one volunteer are shown in Figure 2. All images shown in the Results section are oriented according to radiological convention; the right side of the images corresponds to the left side of the brain. Consistent with the known physiological expectations, the gray matter exhibits a higher $\mathrm{rCBF}$ when compared to that of the white matter. Furthermore, regional CBF of gray matter and white matter for all volunteers are summarized in Table 2. In accordance with the reported values in the literature, a mean $\mathrm{rCBF}$ of $68.1 \pm 9.5 \mathrm{~mL} / 100 \mathrm{~g} /$ minute was obtained for the gray matter, while $26.7 \pm 5.0 \mathrm{~mL} / 100 \mathrm{~g} /$ minute was obtained for the white matter.

Figure 3 shows anatomical images from one patient who exhibited a complete occlusion of the left internal carotid artery demonstrated by the digital subtraction angiogram. The patient had a prior cerebral ischemia, resulting in cerebral infarction, which was exhibited as hypointensity in the T1-weighted images (Figs. 3a-c) and hyperintensity in the T2-weighted images (Figs. 3d-f). Petechial hemorrhage was also observed (arrow) in the cortical rim.

The co-registered MR (Fig. 4a) and PET (Fig. 4b) CBF maps from the same subject shown in Figure 3 are shown in Figure 4. A similar range of CBF was observed for both the PET and MR estimates. Because this patient suffered from a complete occlusion of the left carotid artery, an asymmetry in the CBF was observed between the two hemispheres with the ipsilateral (left) hemisphere exhibiting a lower CBF when compared to the contralateral (right) hemisphere. In addition, a rather low CBF was seen in the infarct region.

Finally, MR estimated CBF were correlated to those obtained from PET measurements and the result of each patient is shown in Figure 5. While a highly linear relationship between PET and MR was obtained for each patient ( $\mathrm{r}$ values ranging between $0.70-0.91$ ) when the proposed correction algorithm was not employed, the slopes of the linear regression lines varied among subjects (between 0.65-1.58) (Fig. 5). As a result, when data from all patients were analyzed as a group, no clear relationship $(r=0.41)$ between MR and PET estimates of CBF was obtained (Fig. 6a). In contrast, a substantial improvement was obtained in the relationship between PET and MR estimates of CBF after the application of the VOF correction scheme (Fig. 6b) with a slope of 1.02 and $r=0.80$. 


\section{DISCUSSION}

In this study, patients with angiographically proven unilateral carotid artery occlusion were recruited for obtaining CBF measurements with both MR and PET so that the accuracy of MR measured CBF with a known cerebrovascular disease could be assessed. The choice of unilateral carotid artery occlusion patients for this study was primarily determined based on two reasons. First, alterations of cerebral hemodynamics are commonly associated with cerebrovascular disease $(9,22,23)$. Many investigators have demonstrated a range of alterations in $\mathrm{CBF}$ and $\mathrm{CBV}$ in the hemisphere ipsilateral to the stenostic and/or occluded vessel, presumably reflecting variable degrees of collateral flow $(9,22)$. Therefore, a physiologically relevant $\mathrm{CBF}$ range was available in this group of patients, providing a good test paradigm to assess the accuracy of MR estimated CBF when compared to that obtained via PET. Second, because unilateral carotid artery occlusion is a chronic disease with rather stable cerebral hemodynamics, it allows comparisons of sequentially measured CBF via different imaging modalities without the concerns of rapid changes that occur in acute ischemic stroke patients.

Despite the fact that results obtained from the volunteer studies (mean $\mathrm{CBF}=68.1 \pm 9.5$ $\mathrm{mL} / 100 \mathrm{~g} /$ minute for gray matter and $\mathrm{CBF}=26.7 \pm 5.0 \mathrm{~mL} / 100 \mathrm{~g} /$ minute for white matter) were consistent with the reported values in the literature (13) and a linear relationship existed for each patient when MR measured CBF is correlated to that obtained via PET (Fig. 5), no clear relationship was observed in the patient group when the MR measured CBF was correlated to that obtained by PET (Fig. 6a). In addition, even though all subjects (including both patients and normal volunteers) received an identical dose of the contrast agent $(0.1$ $\mathrm{mmol} / \mathrm{kg}$ ), a large variability in the estimates of the areas of AIF and VOF (Table 1) was observed in the patient group. A comparable variability in the measure of the area of AIF was observed in the volunteer group when compared to the patient group, yet the observed variability in the estimates of the VOF was much smaller for the volunteer group (Table 1). With all of the above findings, several potential conclusions can be drawn. First, since a linear relationship between MR and PET measured CBF was observed for each patient, a scale factor may exist for each individual patient to correct for the observed variability of MR estimated CBF among patients. Second, the experimentally measured AIF may be partial volume with the surrounding brain parenchyma because the small caliber of the middle cerebral artery and the limited spatial resolution of the EPI sequences. As pointed out recently by van Osch, et al (24), partial volume effects between the AIF and the adjacent brain parenchyma may cause a non-linear behavior between the contrast-induced signal loss and the concentration of contrast. As a result, the areas of AIF may be underestimated in both the patient and the volunteer groups, leading to variability in the CBF estimates. The extent of variability in the measures of $\mathrm{CBF}$ will lie largely on the degree of partial volume effects. Therefore, in order to obtain a more accurate estimate of CBF, the effects of partial volume on the AIF need to be corrected. Thanks to the large caliber of the superior sagittal sinus, the potential partial volume effects imposed by the limited spatial resolution of the EPI sequences can be substantially minimized with the measurements of the area of VOF (Table 1). In addition, since the areas of AIF and VOF should be identical, a correction factor, in theory, can be obtained based on the ratio of the areas of AIF and VOF (AIF/VOF) 
of each patient. Thus, the errors caused by the AIF in the estimated of CBF can be minimized. Unfortunately, this approach fails to improve the relationship between MR and PET measured CBF for the patient studies (data not shown).

Although many possibilities may explain why this approach fails to improve the relationship, one of the most plausible explanations lies within the assumptions made by Zierler (25) in deriving the tracer kinetics. It is assumed that no tracers leave the ROI until all tracers have arrived. Since the slice thickness of the MR images is rather thin and an intravascular contrast agent is employed, this assumption made by Zierler (25) may no longer be valid for MR imaging approaches. In other words, the maximal height of the normalized residue function will not be equal to one because some of the tracers may have left prior to the arrival of all tracers in the ROI. As a result, inaccuracy, as well as variability, in the measurements of $\mathrm{CBF}$ may occur. However, this confounding factor may be negligible in the normal volunteer group since the fractional cardiac output to the brain is anticipated to be rather stable. In contrast, a compromised and variable fractional cardiac output to the brain is highly likely in the patient group, leading to a substantial dispersion of the bolus of contrast agent. Therefore, even though the ratio of AIF/VOF can potentially minimize the errors caused by the partial volume of the AIF in the estimates of CBF, it cannot correct for the dispersion induced by the compromised fractional cardiac output to the brain. In this study, instead of using the ratio of AIF/VOF of each patient to correct for the MR measured $\mathrm{CBF}$, a $\mathrm{CF}$ equal to the ratio of $\mathrm{VOF} / \mathrm{VOF}_{\text {mean }}$ is employed for each individual patient, where the $\mathrm{VOF}_{\text {mean }}$ is the mean area of the VOF obtained from the volunteer group. In other words, the fractional cardiac output to the brain is forced to be identical for both the patient and the volunteer groups and thereby minimizes the potential confounding factors induced by the partial volume effects of the AIF, as well as the bolus dispersion, in the patient group.

With the experimentally derived CF for each patient, a substantial improvement is observed not only in the linearity $(r=0.8)$ but also the slope of the linear regression line $(1.02)$, indicating that, potentially, a more accurate estimate of CBF across a range of physiologically relevant $\mathrm{CBF}$ can be achieved with MR when the proposed correction algorithm is employed (Fig. 6b). Nevertheless, the proposed CBF correction scheme embodies two major assumptions. First, the total concentration of contrast agent during the first pass of the contrast agent needs to be comparable among the subjects, including both volunteers and patients, so that the contrast-induced total signal changes in a voxel, which is $100 \%$ blood, will be identical. Thus, $\mathrm{VOF}_{\text {mean }}$ obtained from the volunteer studies can be used to correct for the CBF estimates in the patients. However, this assumption may not always be valid given the fact that the fractional cardiac output to the brain may vary between subjects. This may explain in part the observed variability in the estimates of the areas of VOF even in the normal volunteers. Second, the contrast-induced signal reduction during the first pass of the contrast agent needs to be above the noise level so that the relationship between the induced signal changes and the concentration of contrast agent remains linear. In this study, a single dose of contrast agent is employed for both the volunteer and patient studies. Hence, it is unlikely that the effects of contrast agent will result in signal reduction lower than the noise level. However, this assumption may limit the applicability of the proposed approach for a double dose study where the effects of contrast 
agent may no longer behave linearly with the induced signal changes. Nevertheless, since a gradient-echo EPI sequence is used to augment the sensitivity in detecting contrast agent induced signal changes, a single dose provides sufficient signal-to-noise ratio (SNR) for the estimates of CBF in this study.

The non-zero y-intercept of the linear regression line shown in Figure $6 \mathrm{~b}$ could be accounted for by the following explanations. First, the effects of large vessels are present in MR images but not in the PET. It has been suggested that a gradient-echo EPI sequence is more sensitive to large vessel effects when compared to a spin-echo EPI sequence, leading to a larger than anticipated signal reduction in the presence of contrast agent (26). As a result, the ROI analysis employed in our study for CBF measurements may include information from large vessels, particularly for the estimates of gray matter CBF, causing an overestimation of CBF. As shown in Figure 2, all the cortical vessels exhibit a high CBF. Although the effects of large vessels can be minimized with the employment of a spin-echo EPI sequence (26), the resulting lower SNR and the need for administering a double dose of contrast agent have prevented us from using a spin echo EPI sequence. Alternatively, a threshold based on the estimates of CBV can be used to remove large vessel effect. More studies will be needed to further determine the extent of the effects of large vessels on the relationship between MR and PET estimates of CBF. Second, the method by which the contrast agent was administered to the patient and volunteer groups differed. In the patient group, the contrast agent was administered by manual injection, while a power injector was employed for the volunteer studies. This discrepancy may potentially induce a systematical error for the proposed correction approach. Third, since $\mathrm{H}_{2} 15 \mathrm{O}$ was used as a tracer for obtaining CBF measurements with PET, it has been shown that an underestimation of CBF may occur for high CBF values (27). As a result, the MR measured CBF is higher than that of PET. Finally, partial volume effects in the estimates of VOF may still be present, particularly in the patient population. Nevertheless, the partial volume effect can be further minimized by acquiring images with a smaller field-of-view. With the improved gradient capability in most of the clinical scanners, this should be easily achievable and can potentially further minimize the effects of partial volume in the estimates of VOF.

In conclusion, we have demonstrated that a more consistent estimate of CBF can be obtained with MR under a pathophysiological condition by employing a correction factor based on the area of VOF. After globally scaling the CBF based on the experimentally derived CF, a substantial improvement is observed in the correlation between the MR and PET estimated CBF. A slope of 1.02 and $r=0.8$ is obtained for the linear regression line, suggesting that this approach provides a more consistent estimate of CBF. However, the nonzero y-intercept still limits the accuracy of this technique especially at lower CBF values. Further work will be needed to investigate the source(s) that cause the non-zero y-intercept.

\section{Acknowledgments}

Contract grant sponsor: National Institutes of Health; Contract grant number: NS37312 and NSO6833i. 


\section{REFERENCES}

1. Powers WJ. Cerebral hemodynamics in ischemic cerebrovascular disease. Ann Neurol. 1991; 29:231-240. [PubMed: 2042939]

2. Rosen BR, Belliveau JW, Vevea JM, Brady TJ. Perfusion imaging with NMR contrast agents. Magn Reson Med. 1990; 14:249-265. [PubMed: 2345506]

3. Rosen BR, Belliveau JW, Aronen HJ, et al. Susceptibility contrast imaging of cerebral blood volume: human experience. Magn Reson Med. 1991; 22:293-299. [PubMed: 1812360]

4. Warach S, Li W, Ronthal M, Edelman RR. Acute cerebral ischemia: evaluation with dynamic contrast-enhanced MR imaging and MR angiography. Radiology. 1992; 182:41-47. [PubMed: 1727307]

5. Hamberg LM, Macfarlane R, Tasdemiroglu E, et al. Measurement of cerebrovascular changes in cats after transient ischemia using dynamic magnetic resonance imaging. Stroke. 1993; 24:444-450. [PubMed: 8446981]

6. Guckel F, Brix G, Rempp K, Deimling M, Rother J, Georgi M. Assessment of cerebral blood volume with dynamic susceptibility contrast enhanced gradient-echo imaging. J Comput Assist Tomogr. 1994; 18:344-351. [PubMed: 8188897]

7. Aronen HJ, Gazit IE, Louis DN, et al. Cerebral blood volume maps of gliomas: comparison with tumor grade and histologic findings. Radiology. 1994; 191:41-51. [PubMed: 8134596]

8. Pan Y, Lo EH, Matsumoto K, Hamberg L, Jiang H. Quantitative and dynamic MRI of neuroprotection in experimental stroke. J Neurol Sci. 1995; 131:128-134. [PubMed: 7595637]

9. Lythgoe DJ, Ostergaard L, William SC, et al. Quantitative perfusion imaging in carotid artery stenosis using dynamic susceptibility contrast-enhanced magnetic resonance imaging. Magn Reson Imaging. 2000; 18:1-11. [PubMed: 10642097]

10. Ostergaard L, Weisskoff RM, Chesler DA, Gyldensted C, Rosen BR. High resolution measurement of cerebral blood flow using intra-vascular tracer bolus passages. Part I: mathematical approach and statistical analysis. Magn Reson Med. 1996; 36:715-725. [PubMed: 8916022]

11. Ostergaard L, Sorensen AG, Kwong KK, Weisskoff RM, Gyldensted C, Rosen BR. High resolution measurement of cerebral blood flow using intravascular tracer bolus passages. Part II: experimental comparison and preliminary results. Magn Reson Med. 1996; 36:726-736. [PubMed: 8916023]

12. Ostergaard L, Smith DF, Vestergaard-Poulsen P, et al. Absolute cerebral blood flow and blood volume measured by magnetic resonance imaging bolus tracking: comparison with positron emission tomography values. J Cereb Blood Flow Metab. 1998; 18:425-432. [PubMed: 9538908]

13. Ostergaard L, Johannsen P, Host-Poulsen P, et al. Cerebral blood flow measurements by magnetic resonance imaging bolus tracking: comparison with $[(15) \mathrm{O}] \mathrm{H} 2 \mathrm{O}$ positron emission tomography in humans. J Cereb Blood Flow Metab. 1998; 18:935-940. [PubMed: 9740096]

14. Derdeyn CP, Yundt KD, Videen TO, Carpenter DA, Grubb RL, Powers WJ. Increased oxygen extraction fraction is associated with prior ischemic events in patients with carotid occlusion. Stroke. 1998; 29:754-758. [PubMed: 9550507]

15. Grubb RL Jr, Derdeyn CP, Fritsch SM, et al. Importance of hemodynamic factors in the prognosis of symptomatic carotid occlusion. JAMA. 1998; 280:1055-1060. [PubMed: 9757852]

16. Raichle ME, Martin WR, Herscovitch P, Mintun MA, Markham J. Brain blood flow measured with intravenous H2(15)O. II. Implementation and validation. J Nucl Med. 1983; 24:790-798. [PubMed: 6604140]

17. Herscovitch P, Markham J, Raichle ME. Brain blood flow measured with intravenous H2(15)O. I. Theory and error analysis. J Nucl Med. 1983; 24:782-789. [PubMed: 6604139]

18. Videen TO, Perlmutter JS, Herscovitch P, Raichle ME. Brain blood volume, flow, and oxygen utilization measured with $15 \mathrm{O}$ radiotracers and positron emission tomography: revised metabolic computations. J Cereb Blood Flow Metab. 1987; 7:513-516. [PubMed: 3497165]

19. Welch MJ, Kilbourn MR, Green MA. Radiopharmaceuticals labeled with short-lived positronemitting radionuclides. Radioisotopes. 1985; 34:170-179. [PubMed: 3892598] 
20. Talairach, J.; Tournoux, P. Co-planar stereotaxic atlas of the human brain: 3-dimensional proportional system: an approach to cerebral imaging. Stuttgart and New York: G: Thieme and Thieme Medical Publishers; 1988. p. 9-18.

21. Woods RP, Cherry SR, Mazziotta JC. Rapid automated algorithm for aligning and reslicing PET images. J Comput Assist Tomogr. 1992; 16:620-633. [PubMed: 1629424]

22. Powers WJ, Press GA, Grubb RL, Gado M, Raichle ME. The effect of hemodynamically significant carotid artery disease on the hemodynamic status of the cerebral circulation. Ann Intern Med. 1987; 106:27-34. [PubMed: 3491558]

23. Detre JA, Alsop DC, Vives LR, Maccotta L, Teener JW, Raps EC. Noninvasive MRI evaluation of cerebral blood flow in cerebrovascular disease. Neurology. 1998; 50:633-641. [PubMed: 9521248]

24. van Osch MJP, Vonken EPA, Bakker CJG, Viergever MA. Correcting partial volume artifacts of the arterial input function in quantitative cerebral perfusion MRI. Magn Reson Med. 2001; 45:477-485. [PubMed: 11241707]

25. Zierler C. Equations for measuring blood flow by external monitoring of radioisotopes. Circ Res. 1965; 16:309-321. [PubMed: 14270567]

26. Boxerman JL, Bandettini PA, Kwong KK, et al. The intravascular contribution to fMRI signal change: Monte Carlo modeling and diffusion-weighted studies in vivo. Magn Reson Med. 1995; 34:4-10. [PubMed: 7674897]

27. Herscovitch P, Raichle ME, Kilbourn MR, Welch MJ. Positron emission tomographic measurement of cerebral blood flow and permeability-surface area product of water using [15O]water and [11C]butanol. J Cereb Blood Flow Metab. 1987; 7:527-542. [PubMed: 3498732] 

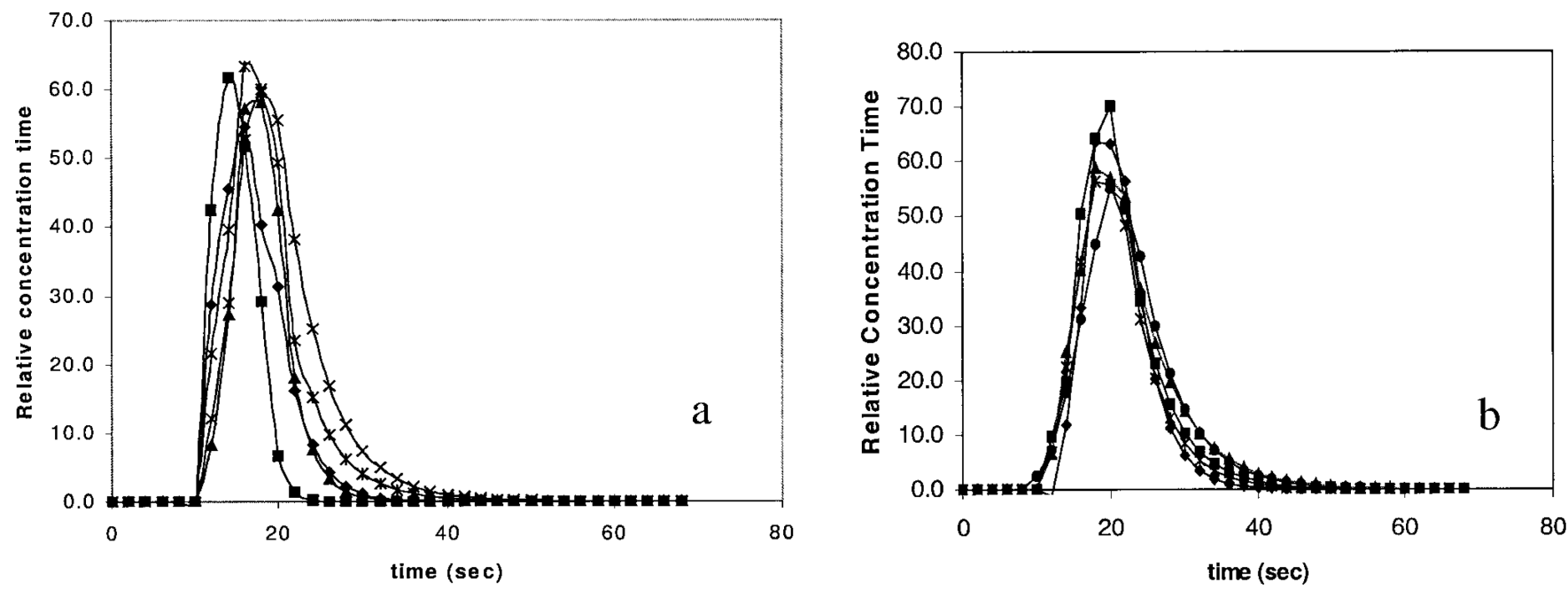

Figure 1.

The concentration time curves for both the AIF (a) and the VOF (b) obtained from the volunteer group. A substantial variability is observed in the AIF compared to the VOF. 


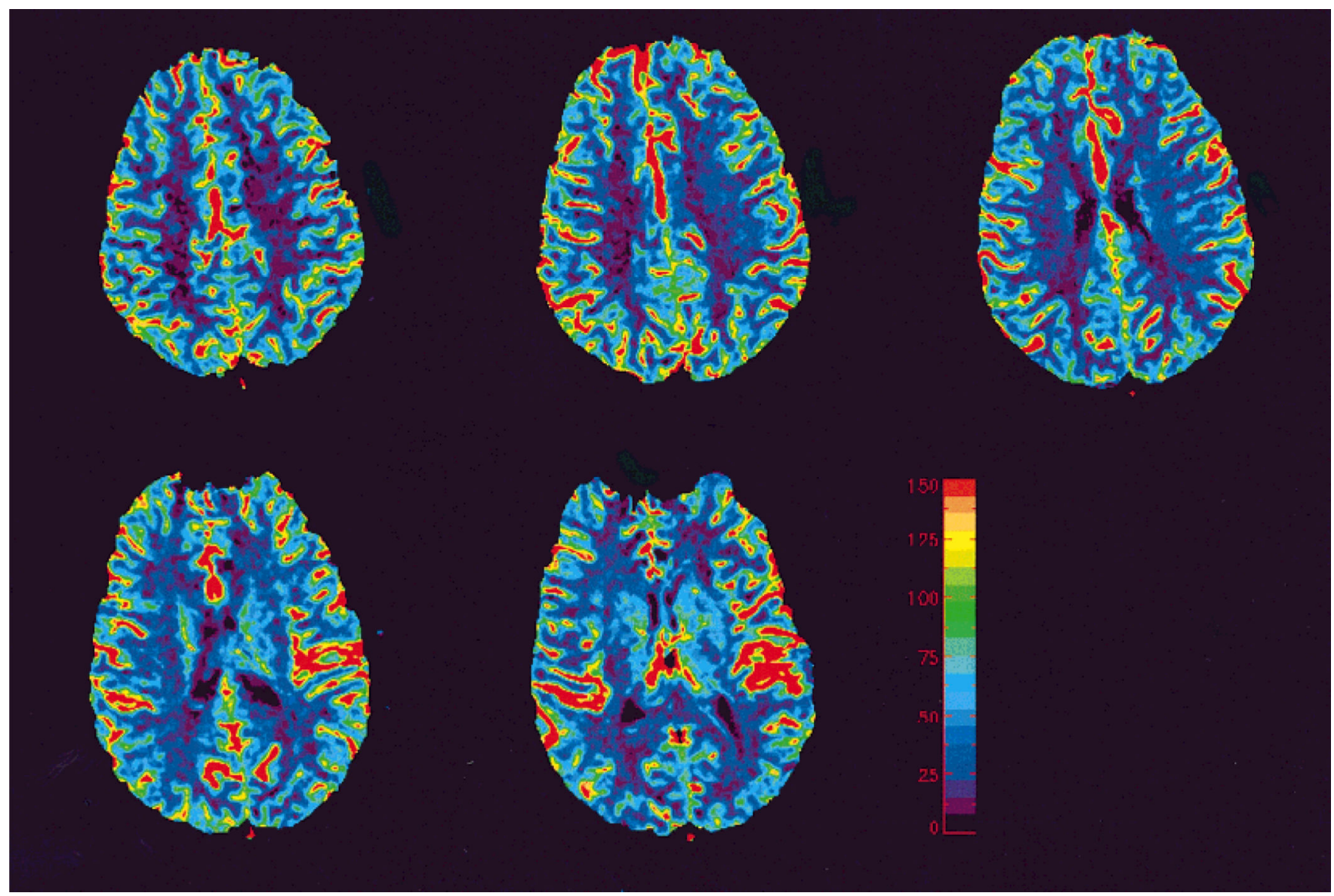

Figure 2.

CBF maps obtained from a healthy volunteer across five contiguous slices. Notice the high CBF values are associated with cortical vessels. 

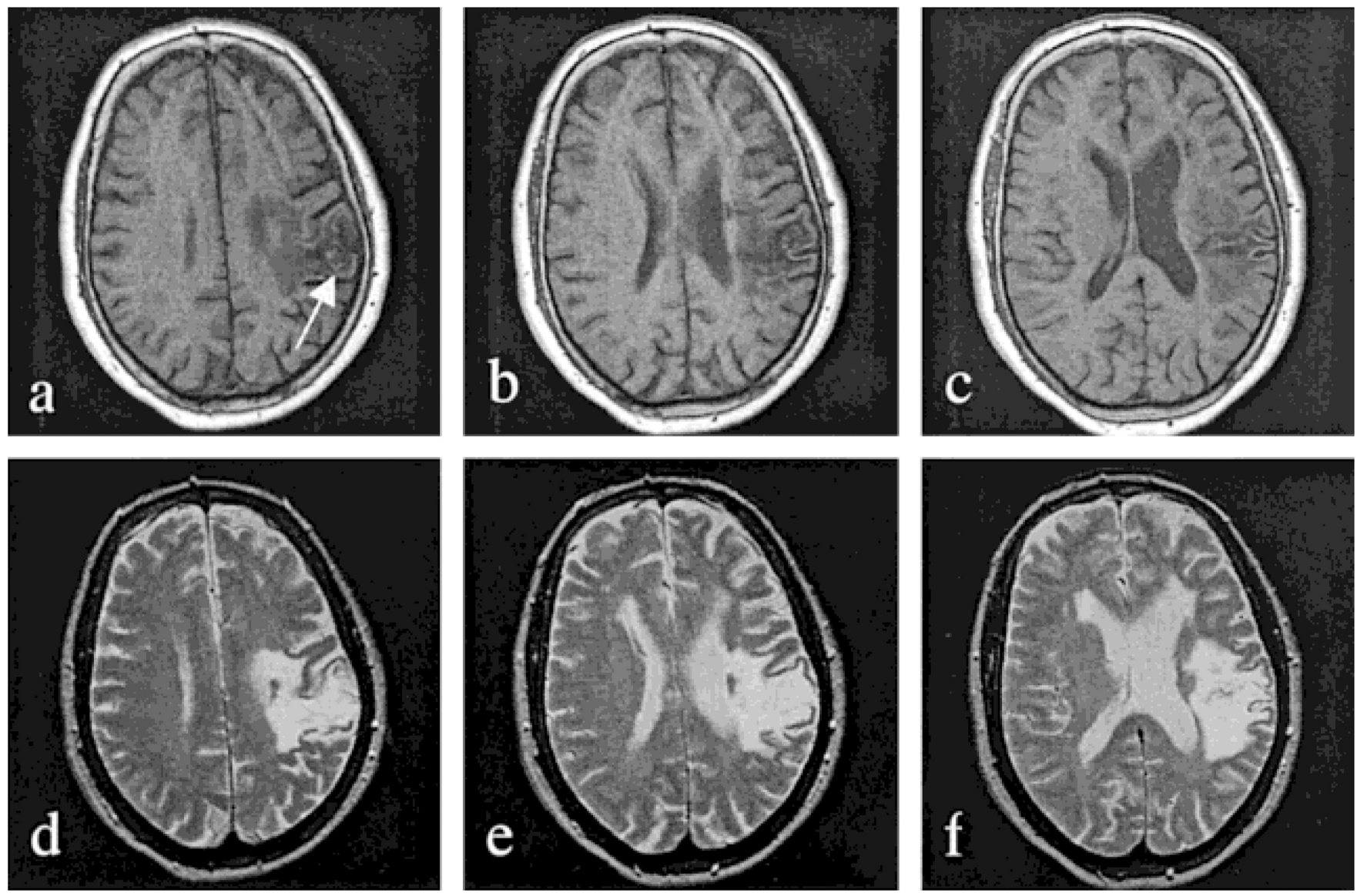

Figure 3.

Representative T1- (a-c) and T2-weighted (d-e) images obtained from a patient with left internal carotid occlusion. 
Figure 4.

A comparison of the co-registered MR (a) and PET (b) CBF maps is shown. 

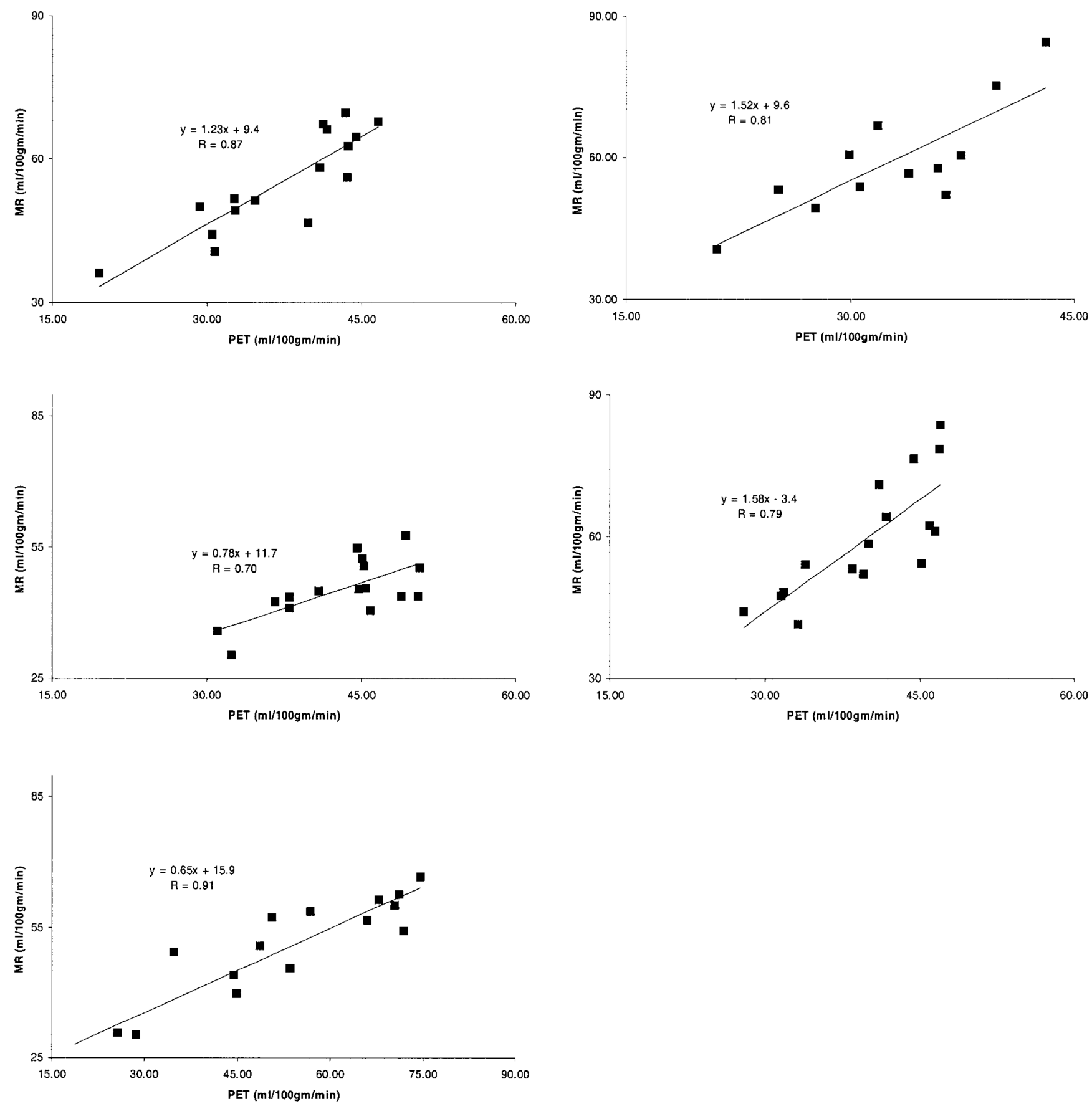

Figure 5.

Relationship between MR measured CBF and that obtained from PET for each patient is shown. Despite the observed linear relationship in each individual patient, the slopes of the linear regression lines vary between patients. 

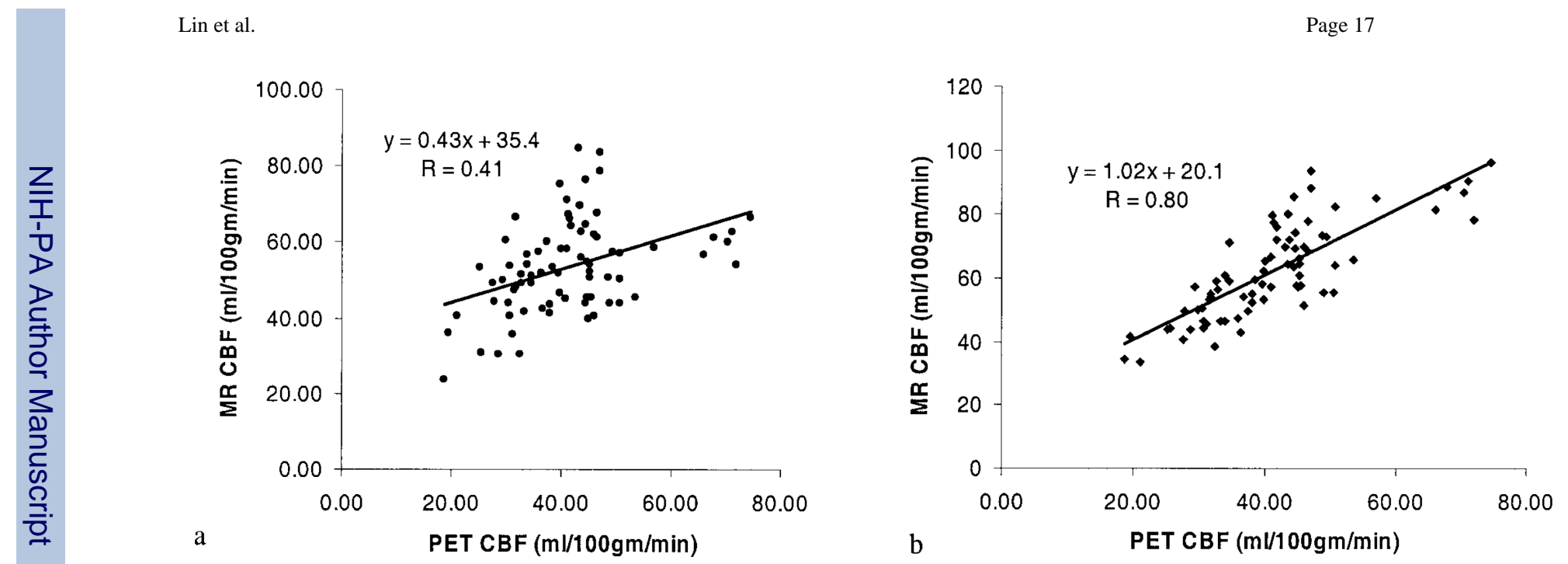

Figure 6.

Relationships between MR estimated CBF and that obtained from PET are shown without (a) and with the proposed correction algorithm (b). 
Table 1

Comparisons Between the Areas of AIF and VOF in the Control and Patient Groups

\begin{tabular}{cccccc}
\hline \multirow{2}{*}{ Subject } & \multicolumn{2}{c}{ Volunteer studies } & & \multicolumn{2}{c}{ Patient studies } \\
\cline { 2 - 3 } \cline { 5 - 6 } \cline { 5 - 6 } & Area of AIF & Area of VOF & & Area of AIF & Area of VOF \\
\hline 1 & 467 & 608 & & 339 & 752 \\
2 & 387 & 670 & & 462 & 944 \\
3 & 451 & 592 & & 441 & 829 \\
4 & 643 & 648 & & 495 & 540 \\
5 & 599 & 751 & & 644 & 734 \\
Mean/SD & $509.4 \pm 107.3^{*}$ & $653.8 \pm 62.6$ & & $476.2 \pm 110.4$ & $759.6 \pm 148.0$ \\
\hline$*$ & & & & & \\
$P<0.02$. & & &
\end{tabular}




\section{Table 2}

Cerebral Blood Flow Measurements in Normal Volunteers

\begin{tabular}{ccc}
\hline Subject & $\begin{array}{c}\text { GM CBF } \\
(\mathbf{m L / 1 0 0} \mathbf{g} / \text { minute })\end{array}$ & $\begin{array}{c}\text { WM CBF } \\
(\mathbf{m L / 1 0 0} \mathbf{g} / \text { minute })\end{array}$ \\
\hline 1 & $76.1 \pm 4.6$ & $33.7 \pm 7.0$ \\
2 & $53.7 \pm 9.15$ & $21.0 \pm 1.9$ \\
3 & $69.8 \pm 6.0$ & $25.5 \pm 6.3$ \\
4 & $64.4 \pm 7.2$ & $23.8 \pm 4.9$ \\
5 & $76.7 \pm 7.5$ & $29.6 \pm 4.9$ \\
Mean/SD & $68.1 \pm 9.5$ & $26.7 \pm 5.0$ \\
\hline
\end{tabular}

J Magn Reson Imaging. Author manuscript; available in PMC 2014 July 18. 